Research Article

Open Access

Special Issue Dedicated to Charles R. Johnson

Macarena Collao, Mario Salas, and Ricardo L. Soto*

\title{
Toeplitz nonnegative realization of spectra via companion matrices
}

https://doi.org/10.1515/spma-2019-0017

Received July 25, 2019; accepted November 26, 2019

Abstract: The nonnegative inverse eigenvalue problem (NIEP) is the problem of finding conditions for the existence of an $n \times n$ entrywise nonnegative matrix $A$ with prescribed spectrum $\Lambda=\left\{\lambda_{1}, \ldots, \lambda_{n}\right\}$. If the problem has a solution, we say that $\Lambda$ is realizable and that $A$ is a realizing matrix. In this paper we consider the NIEP for a Toeplitz realizing matrix $A$, and as far as we know, this is the first work which addresses the Toeplitz nonnegative realization of spectra. We show that nonnegative companion matrices are similar to nonnegative Toeplitz ones. We note that, as a consequence, a realizable list $\Lambda=\left\{\lambda_{1}, \ldots, \lambda_{n}\right\}$ of complex numbers in the left-half plane, that is, with $\operatorname{Re} \lambda_{i} \leq 0, i=2, \ldots, n$, is in particular realizable by a Toeplitz matrix. Moreover, we show how to construct symmetric nonnegative block Toeplitz matrices with prescribed spectrum and we explore the universal realizability of lists, which are realizable by this kind of matrices. We also propose a Matlab Toeplitz routine to compute a Toeplitz solution matrix.

Keywords: Toeplitz nonnegative inverse eigenvalue problem, Unit Hessenberg Toeplitz matrix, Symmetric nonnegative block Toeplitz matrix, Universal realizability

MSC: $15 \mathrm{~A} 29,15 \mathrm{~A} 18$

Dedicated with admiration and special thanks to Charles R. Johnson.

\section{Introduction}

The nonnegative inverse eigenvalue problem (hereafter NIEP) is the problem of finding necessary and sufficient conditions for a list $\Lambda=\left\{\lambda_{1}, \lambda_{2}, \ldots, \lambda_{n}\right\}$ of complex numbers to be the spectrum of an $n \times n$ entrywise nonnegative matrix . If there exists a nonnegative matrix $A$ with spectrum $\Lambda$, we say that $\Lambda$ is realizable and that $A$ is a realizing matrix. The NIEP has been completely solved for $n=3$ [8] and for $n=4$ [10], and independently in [21]. For $n \geq 5$ the NIEP remains unsolved, although for $n=5$ with $\sum_{i=1}^{n} \lambda_{i}=0$, the problem was solved in [6]. In this paper we consider the NIEP for Toeplitz realizing matrices. An $n \times n$ Toeplitz matrix

Macarena Collao: Departamento de Matemáticas, Universidad Católica del Norte, Casilla 1280, Antofagasta, Chile, E-mail: mcollao02@ucn.cl

Mario Salas: Departamento de Matemáticas, Universidad Católica del Norte, Casilla 1280, Antofagasta, Chile, E-mail: msalas@ucn.cl

*Corresponding Author: Ricardo L. Soto: Departamento de Matemáticas, Universidad Católica del Norte, Casilla 1280, Antofagasta, Chile, E-mail: rsoto@ucn.cl 
is a matrix of the form

$$
T=\left[\begin{array}{ccccc}
t_{0} & t_{1} & t_{2} & \cdots & t_{n-1} \\
t_{-1} & t_{0} & t_{1} & \ddots & \vdots \\
t_{-2} & t_{-1} & \ddots & \ddots & t_{2} \\
\vdots & \ddots & \ddots & \ddots & t_{1} \\
t_{-n+1} & \cdots & t_{-2} & t_{-1} & t_{0}
\end{array}\right] .
$$

$T$ is circulant if $t_{-i}=t_{n-i}, i=1,2, \ldots, n-1$.

It is well known that the inverse eigenvalue problem (IEP) is always solvable for Toeplitz matrices: a Toeplitz circulant matrix $T=F^{-1} D F$ can always be obtained with complex spectrum $\Lambda=\left\{\lambda_{1}, \ldots, \lambda_{n}\right\}$, where $F$ is the matrix of the discrete Fourier transform and $D=\operatorname{diag}\left\{\lambda_{1}, \ldots, \lambda_{n}\right\}$. In contrast to this result, we are interested in the nonnegativity property of a realizing matrix. In this work we show, under certain conditions, that a list of complex numbers is the spectrum of a Toeplitz nonnegative matrix, which is not necessarily circulant. In [9] the authors prove that an $n \times n$ complex nonderogatory matrix is similar to a unique unit upper Hessenberg Toeplitz matrix, and that every $n \times n$ complex matrix with $n \leq 4$ is similar to a Toeplitz matrix. As far as we know, this paper is the first work which addresses the nonnegative Toeplitz realization of spectra. Although Toeplitz matrices are persymmetric, the NIEP for persymmetric matrices (see $[5,19]$ ) and the NIEP for Toeplitz matrices are different. For example, the list $\Lambda=\{6,5,1\}$ is the spectrum of the nonnegative persymmetric matrix

$$
P=\left[\begin{array}{lll}
3 & 0 & 2 \\
0 & 6 & 0 \\
2 & 0 & 3
\end{array}\right] .
$$

However, it is easy to check that $\Lambda$ cannot be the spectrum of a nonnegative matrix with diagonal entries 4,4 , 4. In this paper we give sufficient conditions for the existence and construction of a nonnegative Toeplitz matrix with prescribed spectrum.

A real matrix $A=\left[a_{i j}\right]_{i, j=1}^{n}$ is said to have constant row sums if all its rows sum up to the same constant $\alpha$, i.e., $\sum_{j=1}^{n} a_{i j}=\alpha, i=1, \ldots, n$.

The set of all real matrices with constant row sums equal to $\alpha$ is denoted by $\mathcal{e} \mathcal{S}_{\alpha}$. It is clear that $\mathbf{e}=$ $[1,1, \ldots, 1]^{T}$ is an eigenvector of any matrix in $\mathcal{C} \mathcal{S}_{\alpha}$, corresponding to the eigenvalue $\alpha$. The relevance of matrices with constant row sums is due to the well known fact that if $\lambda_{1}$ is the desired Perron eigenvalue, then the problem of finding a nonnegative matrix with spectrum $\Lambda=\left\{\lambda_{1}, \ldots, \lambda_{n}\right\}$ is equivalent to the problem of finding a nonnegative matrix in $\mathcal{C S}_{\lambda_{1}}$ with spectrum $\Lambda$.

The paper is organized as follows: In Section 2, we show that nonnegative companion matrices are similar to nonnegative Toeplitz ones. As a consequence, a realizable list of complex numbers in the left-half plane, that is, a list $\Lambda=\left\{\lambda_{1}, \ldots, \lambda_{n}\right\}$ with $\operatorname{Re} \lambda_{i} \leq 0, i=2, \ldots, n$, which includes real and complex lists of Suleimanova [20] and Šmigoc [14] type, is in particular realizable by a Toeplitz matrix. In Section 3, we give sufficient conditions for the existence of a symmetric nonnegative block Toeplitz matrix with prescribed Jordan canonical form. We also give examples to illustrate the results. Finally, we implement a Matlab Toeplitz routine for computing the desired Toeplitz matrix, which is introduced in the Appendix, Section 4.

\section{Companion matrices are similar to Toeplitz ones}

In this Section, we show that an $n \times n$ nonnegative companion matrix $\mathcal{C}$ is similar to a nonnegative Toeplitz matrix $T$ by a lower triangular similarity. This result, interesting in itself, gives rise to a sufficient condition for 
the existence and construction of a nonnegative Toeplitz matrix with prescribed complex spectrum. It allows us to show that a list $\Lambda=\left\{\lambda_{1}, \ldots, \lambda_{n}\right\}$ of complex numbers in the left half-plane, that is, with $\operatorname{Re} \lambda_{i} \leq 0$, $i=2, \ldots, n$, is in particular realizable by a Toeplitz matrix.

Theorem 2.1. Let

$$
\mathcal{C}=\left[\begin{array}{ccccc}
0 & 1 & 0 & \cdots & 0 \\
0 & 0 & \ddots & \ddots & \vdots \\
\vdots & \vdots & \ddots & \ddots & 0 \\
0 & 0 & \cdots & 0 & 1 \\
c_{n} & c_{n-1} & \cdots & c_{2} & c_{1}
\end{array}\right]
$$

be the companion matrix of the polynomial $p(x)=x^{n}-\sum_{i=1}^{n} c_{i} x^{n-i}$. Then $\mathcal{C}$ is similar to a unit lower Hessenberg Toeplitz matrix $T$.

Proof. First, we shall assume that $c_{1}=0$. Define the $n \times n$ real matrix

$$
S=\left[\begin{array}{ccccccc}
1 & 0 & \cdots & \cdots & \cdots & \cdots & 0 \\
0 & 1 & \ddots & & & & \vdots \\
s_{21} & 0 & \ddots & \ddots & & & \vdots \\
s_{31} & s_{22} & \ddots & \ddots & \ddots & & \vdots \\
s_{41} & s_{32} & \ddots & \ddots & \ddots & \ddots & \vdots \\
\vdots & \ddots & \ddots & \ddots & 0 & 1 & 0 \\
s_{(n-1) 1} & \cdots & s_{4(n-4)} & s_{3(n-3)} & s_{2(n-2)} & 0 & 1
\end{array}\right],
$$

where, for convenience, $s_{i j}$ is the entry on the diagonal $(j+i, j), i=2,3, \ldots,(n-1) ; j=1,2, \ldots,(n-i)$. The entries of $S^{-1}$ can be obtained in a straightforward manner. In fact, since $\operatorname{det} S=1$, then $S^{-1}=\operatorname{adj} S$, and the entries $(i, j)$ of $S^{-1}$ are given by

$$
\left[S^{-1}\right]_{i, j}=(-1)^{i+j} \operatorname{det} \widehat{S}_{j i},
$$

where $\widehat{S}_{j i}$ is the matrix $S$ without the row $j$ and the column $i$. We utilize MATLAB notation: for $i<j$, we let $i: j$ denote the set $\{x \in \mathbb{Z}: i \leq x \leq j\}$ and $A(i: j, k: l)$ denote the submatrix of $A$ whose rows and columns are indexed by $i: j$ and $k: l$, respectively. Then, $\operatorname{det} \widehat{S}_{j i}=\operatorname{det} S(j+1: i, j: i-1), i=3, \ldots n, j=1, \ldots, i-2$. Thus, the formula (3) becomes

$$
\left[S^{-1}\right]_{i, j}=(-1)^{i+j} \operatorname{det} S(j+1: i, j: i-1), i=3, \ldots n, j=1, \ldots, i-2 .
$$

We consider the $n \times n$ matrix 


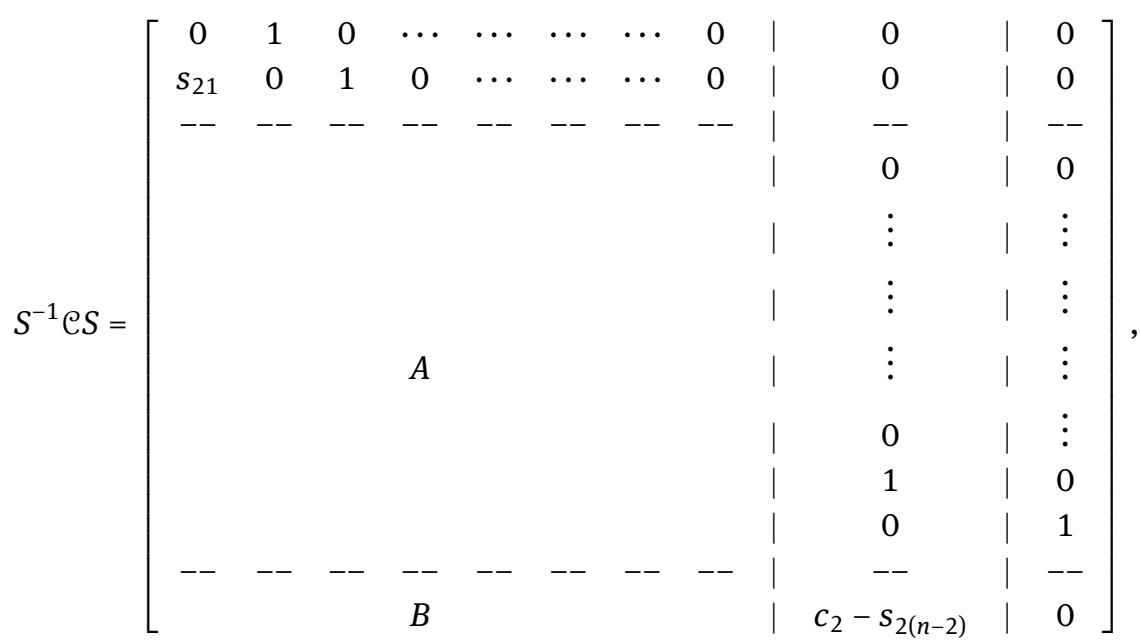

where the entries on the block $A$ are given by

$$
\left[S^{-1} \mathcal{C} S\right]_{i j}=s_{(i-j+1) j}+\sum_{k=0}^{i-j+1}(-1)^{i-j-k+3} \operatorname{det} S(j+k: i, j+k-1: i-1) s_{k j},
$$

$i=3, \ldots, n-1, j=1, \ldots, n-2$, with $s_{0 j}=1, s_{1 j}=0, s_{p j}=0$ for $p<0, \operatorname{det} S(1: i, 0: i-1)=0$ and $\operatorname{det} S(h: g, h-1: g-1)=0$ if $h>g$, while the entries on the block $B$ are given by

$$
\left[S^{-1} \mathcal{C} S\right]_{n j}=\sum_{k=0}^{n-j+1}\left((-1)^{n-j+3-k} \operatorname{det} S(j+k: n, j+k-1: n-1) s_{k j}+c_{k} S_{(n-j-k+1) j}\right),
$$

$i=n, j=1, \ldots, n-2$, with $s_{0 j}=1, s_{1 j}=0, c_{l}=0$ for $l \leq 1$ and $\operatorname{det} S(i: j ; k: l)=0$ if $i>j$. Then $S^{-1}$ eS has the form

$$
\left[\begin{array}{cccccccc}
0 & 1 & 0 & \cdots & \cdots & \cdots & \cdots & 0 \\
s_{21} & \ddots & \ddots & \ddots & & & \vdots \\
s_{31} & s_{22}-s_{21} & \ddots & \ddots & \ddots & & & \vdots \\
s_{41}-s_{21} s_{22} & s_{32}-s_{31} & s_{23}-s_{22} & \ddots & \ddots & \ddots & & \vdots \\
\left(s_{51}-s_{21} s_{32}\right. & \left(s_{21} s_{23}-s_{41}\right. & s_{33}-s_{32} & \ddots & \ddots & \ddots & \ddots & \vdots \\
\left.-s_{31} s_{23}\right) & \left.-s_{22} s_{23}+s_{42}\right) & \ddots & \ddots & \ddots & \ddots & \ddots & 0 \\
\vdots & \ddots & \ddots & \ddots & s_{3(n-3)}-s_{3(n-4)} & s_{2(n-2)}-s_{2(n-3)} & \ddots & 1 \\
\vdots & \vdots & \vdots & \cdots & \cdots & c_{3}-s_{3(n-3)} & c_{2}-s_{2(n-2)} & 0
\end{array}\right] \text {, }
$$

and it will be a Toeplitz matrix if all the entries in each diagonal under the main diagonal are equal. Then, the entries $s_{i j}$ of $S$ are obtained by equating the entries in each diagonal under the main diagonal of $S^{-1} \mathrm{CS}$. Observe that on each one of these diagonals, each new entry from left to right contains exactly one new unknown. Then, the corresponding linear system always has a unique solution. Thus, our construction is unique. If $\operatorname{tr}(\mathcal{C})=c_{1} \neq 0$, then by taking

$$
S=\left[\begin{array}{cccccc}
1 & 0 & \cdots & \cdots & \cdots & 0 \\
s_{11} & 1 & \ddots & & & \vdots \\
s_{21} & s_{12} & \ddots & \ddots & & \vdots \\
s_{31} & s_{22} & \ddots & \ddots & \ddots & \vdots \\
\vdots & \ddots & \ddots & \ddots & \ddots & 0 \\
s_{(n-1) 1} & \cdots & s_{3(n-3)} & s_{2(n-2)} & s_{1(n-1)} & 1
\end{array}\right], T=\left[\begin{array}{ccccc}
t_{1} & 1 & 0 & \cdots & 0 \\
t_{2} & t_{1} & \ddots & \ddots & \vdots \\
\vdots & \ddots & \ddots & \ddots & 0 \\
\vdots & \ddots & \ddots & \ddots & 1 \\
t_{n} & \cdots & \cdots & t_{2} & t_{1}
\end{array}\right],
$$


we also have similarity between $\mathcal{C}$ and $T$.

Remark 2.1. For our purpose, it is enough to prove the similarity between matrices $\mathcal{C}$ and $T$ for the case $\operatorname{tr}(\mathcal{C})=$ 0 . If $\mathcal{C}$ has spectrum $\Lambda=\left\{\lambda_{1}, \lambda_{2}, \ldots, \lambda_{n}\right\}$ with $c_{1}=\operatorname{tr}(\mathcal{C}) \neq 0$, we take the companion matrix $\mathrm{C}^{\prime}$ with spectrum $\Lambda^{\prime}=\left\{\lambda_{1}-\alpha, \lambda_{2}-\alpha, \ldots, \lambda_{n}-\alpha\right\}$, where $\alpha=\frac{1}{n} c_{1}$. Then $\operatorname{tr}\left(\mathcal{C}^{\prime}\right)=0$ and $\mathcal{C}^{\prime}$ is similar to a Toeplitz matrix $T^{\prime}$ with $\operatorname{tr}\left(T^{\prime}\right)=0$. Therefore $T=T^{\prime}+\alpha I$ is the required Toeplitz matrix, which is cospectral to $\mathrm{C}$.

Example 2.1. Let us consider $n=5$ with $c_{1}=0$. Then we have

$$
S=\left[\begin{array}{ccccc}
1 & 0 & 0 & 0 & 0 \\
0 & 1 & 0 & 0 & 0 \\
s_{21} & 0 & 1 & 0 & 0 \\
s_{31} & s_{22} & 0 & 1 & 0 \\
s_{41} & s_{32} & s_{23} & 0 & 1
\end{array}\right], S^{-1}=\left[\begin{array}{ccccc}
1 & 0 & 0 & 0 & 0 \\
0 & 1 & 0 & 0 & 0 \\
-s_{21} & 0 & 1 & 0 & 0 \\
-s_{31} & -s_{22} & 0 & 1 & 0 \\
s_{21} s_{23}-s_{41} & -s_{32} & -s_{23} & 0 & 1
\end{array}\right],
$$

with

$$
S^{-1} \mathcal{C S}=\left[\begin{array}{ccccc}
0 & 1 & 0 & 0 & 0 \\
s_{21} & 0 & 1 & 0 & 0 \\
s_{31} & s_{22}-s_{21} & 0 & 1 & 0 \\
s_{41}-s_{21} s_{22} & s_{32}-s_{31} & s_{23}-s_{22} & 0 & 1 \\
\left(c_{5}+c_{2} s_{31}+c_{3} s_{21}\right. & \left(c_{4}-s_{41}+c_{2} s_{22}\right. & c_{3}-s_{32} & c_{2}-s_{23} & 0 \\
\left.-s_{21} s_{32}-s_{31} s_{23}\right) & \left.+s_{21} s_{23}-s_{22} s_{23}\right) & & &
\end{array}\right] .
$$

If we take $s_{21}=\frac{1}{4} c_{2}, s_{22}=\frac{1}{2} c_{2}, s_{23}=\frac{3}{4} c_{2}, s_{31}=\frac{1}{3} c_{3}, s_{32}=\frac{2}{3} c_{3}$, and $s_{41}=\frac{1}{2} c_{4}+\frac{7}{32} c_{2}^{2}$, then $S^{-1} \mathrm{C} S$ is the Toeplitz matrix

$$
T=\left[\begin{array}{ccccc}
0 & 1 & 0 & 0 & 0 \\
\frac{1}{4} c_{2} & 0 & 1 & 0 & 0 \\
\frac{1}{3} c_{3} & \frac{1}{4} c_{2} & 0 & 1 & 0 \\
\frac{1}{2} c_{4}+\frac{3}{32} c_{2}^{2} & \frac{1}{3} c_{3} & \frac{1}{4} c_{2} & 0 & 1 \\
c_{5}+\frac{1}{6} c_{2} c_{3} & \frac{1}{2} c_{4}+\frac{3}{32} c_{2}^{2} & \frac{1}{3} c_{3} & \frac{1}{4} c_{2} & 0
\end{array}\right]
$$

which is nonnegative if $c_{i}, i=2, \ldots, 5$, are nonnegative.

Theorem 2.2. If in Theorem 2.1 the companion matrix

$$
\mathcal{C}=\left[\begin{array}{ccccc}
0 & 1 & 0 & \cdots & 0 \\
0 & 0 & \ddots & \ddots & \vdots \\
\vdots & \vdots & \ddots & \ddots & 0 \\
0 & 0 & \cdots & 0 & 1 \\
c_{n} & c_{n-1} & \cdots & c_{2} & c_{1}
\end{array}\right]
$$

is real nonnegative, then the corresponding Toeplitz matrix $T$ is also real nonnegative.

Proof. In [11], the author proves the following formula, known as Trudi's formula, for the $n$-th order determinant of a lower Hessenberg Toeplitz matrix

$$
T^{\prime}=\left[\begin{array}{ccccc}
t_{1} & t_{0} & 0 & \cdots & 0 \\
t_{2} & t_{1} & \ddots & \ddots & \vdots \\
\vdots & \ddots & \ddots & \ddots & 0 \\
\vdots & \ddots & \ddots & \ddots & t_{0} \\
t_{n} & \cdots & \cdots & t_{2} & t_{1}
\end{array}\right]
$$


That is,

$$
\operatorname{det} T^{\prime}=\sum_{l_{1}+2 l_{2}+\cdots+n l_{n}=n}\left(\begin{array}{c}
l_{1}+\cdots+l_{n} \\
l_{1}, \ldots, l_{n}
\end{array}\right)\left(-t_{0}\right)^{n-l_{1}-\cdots-l_{n}} t_{1}^{l_{1}} t_{2}^{l_{2}} \cdots t_{n}^{l_{n}},
$$

where $\left(\begin{array}{c}l_{1}+\cdots+l_{n} \\ l_{1}, \ldots, l_{n}\end{array}\right)=\frac{\left(l_{1}+\cdots+l_{n}\right) !}{l_{1} ! \cdots l_{n} !}$ is the multinomial coefficient, and the sum is over the integer partitions of $n$. Let $T=S^{-1}$ CS be the matrix in Theorem 2.1. Since $x I-T$ is also a lower Hessenberg Toeplitz matrix, we may compute, from (8), the characteristic polynomial of our Toeplitz matrix

$$
T=\left[\begin{array}{ccccc}
0 & 1 & 0 & \cdots & 0 \\
t_{2} & 0 & \ddots & \ddots & \vdots \\
\vdots & \ddots & \ddots & \ddots & 0 \\
\vdots & \ddots & \ddots & \ddots & 1 \\
t_{n} & \cdots & \cdots & t_{2} & 0
\end{array}\right]
$$

That is,

$$
\operatorname{det}(x I-T)=\sum_{l_{1}+2 l_{2}+\cdots+n l_{n}=n}\left(\begin{array}{c}
l_{1}+\cdots+l_{n} \\
l_{1}, \ldots, l_{n}
\end{array}\right)\left(-t_{2}\right)^{l_{2}}\left(-t_{3}\right)^{l_{3}} \cdots\left(-t_{n}\right)^{l_{n}} x^{l_{1}} .
$$

Now, we equate the coefficients of characteristic polynomials of $T$ and $\mathcal{C}$ in (1),

$$
\operatorname{det}(x I-\mathcal{C})=x^{n}-c_{1} x^{n-1}-c_{2} x^{n-2}-\cdots-c_{n-k} x^{k}-\cdots-c_{n-1} x-c_{n} .
$$

Then,

$$
-c_{k}=\sum_{2 l_{2}+\cdots+k l_{k}=k}\left(\begin{array}{c}
n-k+l_{2}+\cdots+l_{k} \\
n-k, l_{2}, \ldots, l_{k}
\end{array}\right)\left(-t_{2}\right)^{l_{2}}\left(-t_{3}\right)^{l_{3}} \cdots\left(-t_{k}\right)^{l_{k}} .
$$

Observe that in each coefficient of the sum in (10), the term $t_{k}$, with $l_{1}=n-k, k=2,3, \ldots, n$, appears isolated, that is, $t_{k}$ has exponent 1 , and it is not a factor in a product with other factors $t_{i}, i \neq k$. Then, we may compute $t_{k}$ from (12), as

$$
\begin{aligned}
& t_{k}=\frac{1}{n-k+1}\left(c_{k}+g_{k}\right), \text { where } \\
& g_{k}=\sum_{2 l_{2}+\cdots+(k-2) l_{k-2}=k}\left(\begin{array}{c}
n-k+l_{2}+\cdots+l_{k-2} \\
n-k, l_{2}, \ldots, l_{k-2}
\end{array}\right)\left(-t_{2}\right)^{l_{2}} \cdots\left(-t_{k-2}\right)^{l_{k-2}}
\end{aligned}
$$

is a multivariate polynomial with positive coefficients. For example, for $n=5$ we have: $l_{1}+2 l_{2}+\cdots+5 l_{5}=5$ with

$\begin{array}{ccccc}l_{1} & l_{2} & l_{3} & l_{4} & l_{5} \\ 5 & 0 & 0 & 0 & 0 \\ 3 & 1 & 0 & 0 & 0 \\ 2 & 0 & 1 & 0 & 0 \\ 1 & 2 & 0 & 0 & 0 \\ 1 & 0 & 0 & 1 & 0 \\ 0 & 1 & 1 & 0 & 0 \\ 0 & 0 & 0 & 0 & 1\end{array}$

Then

$$
\begin{aligned}
& \operatorname{det}(x I-T)=x^{5}-4 t_{2} x^{3}-3 t_{3} x^{2}+\left(3 t_{2}^{2}-2 t_{4}\right) x+2 t_{2} t_{3}-t_{5} \\
& \operatorname{det}(x I-\mathcal{C})=x^{5}-c_{1} x^{4}-c_{2} x^{3}-c_{3} x^{2}-c_{4} x-c_{5},
\end{aligned}
$$

and

$$
c_{1}=0 ; c_{2}=4 t_{2} ; c_{3}=3 t_{3} ; c_{4}=2 t_{4}-3 t_{2}^{2} ; c_{5}=t_{5}-2 t_{2} t_{3}
$$


Thus, the first terms $t_{k}$ are computed from (13) as

$$
\begin{aligned}
& t_{2}=\frac{1}{n-1} c_{2}, \quad t_{3}=\frac{1}{n-2} c_{3}, \\
& t_{4}=\frac{1}{n-3} c_{4}+\frac{n-2}{2} t_{2}^{2}, \quad t_{5}=\frac{1}{n-4} c_{5}+(n-3) t_{2} t_{3} . \\
& t_{6}=\frac{1}{n-5} c_{6}-\frac{(n-3)(n-4)}{6} t_{2}^{3}+\frac{n-4}{2} t_{3}^{2}+(n-4) t_{2} t_{4} .
\end{aligned}
$$

Observe from (13), that $t_{k} \geq 0$ if the sum of exponents $l_{2}+\cdots+l_{k-2}$ in $\left(-t_{2}\right)^{l_{2}} \cdots\left(-t_{k-2}\right)^{l_{k-2}}$ is an even number, where one or more $l_{j}, j=2, \ldots, k-2$, can be zero. If the sum of exponents is an odd number, we show that the sum is still nonnegative. In fact, observe that in $t_{k}$, the negative terms of the form $-t_{j}^{l_{j}} t_{m}^{l_{m}}$ with $j l_{j}+m l_{m}=k$ and $l_{j}+l_{m}$ being odd, are dominated by positive terms $t_{j}^{l_{j}} t_{m}^{l_{m}}$, obtained from terms of the form $t_{j} t_{k-j}$. In fact, since

$$
j\left(l_{j}-1\right)+m l_{m}=j l_{j}+m l_{m}-j=k-j,
$$

it is clear that $t_{k-j}$ contains a positive term of the form $t_{j}^{l_{j}-1} t_{m}^{l_{m}}$, and then $t_{j}\left(t_{j}^{l_{j}-1} t_{m}^{l_{m}}\right)=t_{j}^{l_{j}} t_{m}^{l_{m}}$. Now, we show that the coefficient

$$
(n-k+2) \frac{1}{n-k+j+1} \frac{\left(n-k+j+l_{j}-1+l_{m}\right) !}{(n-k+j) !\left(l_{j}-1\right) ! l_{m} !},
$$

of the term $t_{j}^{l_{j}} t_{m}^{l_{m}}$ obtained from $t_{j} t_{k-j}$ is greater than or equal to the coefficient

$$
\frac{1}{n-k+1} \frac{\left(n-k+l_{j}+l_{m}\right) !}{(n-k) ! l_{j} ! l_{m} !}
$$

of the negative term $-t_{j}^{l_{j}} t_{m}^{l_{m}}\left(l_{j}+l_{m}\right.$ being odd). In fact, the term in (14) minus the term in (15) gives

$$
\frac{\left(n-k+l_{j}+l_{m}\right) !}{(n-k+1) !\left(l_{j}-1\right) ! l_{m} !}\left(\frac{(n-k+2)\left(n-k+l_{j}+l_{m}+1\right) \cdots\left(n-k+l_{j}+l_{m}+j-1\right)}{(n-k+2)(n-k+3) \cdots(n-k+j+1)}-\frac{1}{l_{j}}\right)
$$

and

$$
\frac{\left(n-k+l_{j}+l_{m}\right) !}{(n-k+1) !\left(l_{j}-1\right) ! l_{m} !}\left(\frac{\left(n-k+l_{j}+l_{m}+1\right) \cdots\left(n-k+l_{j}+l_{m}+j-1\right)}{(n-k+3) \cdots(n-k+j+1)}-\frac{1}{l_{j}}\right) .
$$

Observe that there are $(j-1)$ consecutive integer factors in the numerator and in the denominator of

$$
\frac{\left(n-k+l_{j}+l_{m}+1\right) \cdots\left(n-k+l_{j}+l_{m}+j-1\right)}{(n-k+3) \cdots(n-k+j+1)},
$$

and since $l_{j} \geq 1$ and $l_{j}+l_{m} \geq 2$, then

$$
\frac{\left(n-k+l_{j}+l_{m}+1\right) \cdots\left(n-k+l_{j}+l_{m}+j-1\right)}{(n-k+3) \cdots(n-k+j+1)}-\frac{1}{l_{j}} \geq 0 .
$$

Thus, the coefficient in (14) dominates the coefficient in (15). For negative terms of the form $-t_{j_{1}}^{l_{j_{1}}} t_{j_{2}}^{l_{j_{2}}} \cdots t_{j_{m}}^{l_{m}}$, with $j_{1} l_{j_{1}}+\cdots+j_{m} l_{j_{m}}=k$, we reduce these terms to terms of the form $-t_{j}^{l_{j}} t_{m}^{l_{m}}$, by replacing the last factor, and then we proceed as before. Thus, the sum in (13) does not contain negative addends and $t_{k}$ is nonnegative.

Example 2.2. From (13) we can express $t_{k}$ in terms of $c_{k}$. For instance,

$$
\begin{aligned}
& t_{6}=\frac{1}{n-5} c_{6}-\frac{(n-3)(n-4)}{6} t_{2}^{3}+\frac{n-4}{2} t_{3}^{2}+(n-4) t_{2} t_{4} \\
& t_{6}=\frac{1}{n-5} c_{6}-\frac{(n-3)(n-4)}{6} t_{2}^{3}+\frac{n-4}{2} t_{3}^{2}+(n-4) t_{2}\left(\frac{1}{(n-3)} c_{4}+\frac{n-2}{2} t_{2}^{2}\right) \\
& t_{6}=\frac{1}{n-5} c_{6}+\frac{(2 n-3)(n-4)}{6(n-1)^{3}} c_{2}^{3}+\frac{n-4}{2(n-2)^{2}} c_{3}^{2}+\frac{n-4}{(n-1)(n-3)} c_{2} c_{4} .
\end{aligned}
$$


As mentioned in the Introduction, it is well known that a list of complex numbers $\Lambda=\left\{\lambda_{1}, \ldots, \lambda_{n}\right\}$ is always the spectrum of a Toeplitz matrix, indeed of a circulant matrix $T=F D F^{-1}$, where $D=\operatorname{diag}\left\{\lambda_{1}, \ldots, \lambda_{n}\right\}$ and $F$ denotes the matrix of the discrete Fourier transform. Now, as a first consequence of Theorem 2.1, we have the following simple result, in which the Toeplitz matrix $T$ is not necessarily circulant. It is a unit lower Hessenberg Toeplitz matrix.

Corollary 2.1. Let $\Lambda=\left\{\lambda_{1}, \lambda_{2}, \ldots, \lambda_{n}\right\}$ be a list of complex numbers. Then $\Lambda$ is the spectrum of an $n \times n$ unit lower Hessenberg Toeplitz matrix, which is not necessarily circulant.

Proof. It is immediate from Theorem 2.1.

In [7, Theorem 3], Laffey and Šmigoc solved the NIEP in the left-half plane. That is, they proved that if $\Lambda=$ $\left\{\lambda_{1}, \ldots, \lambda_{n}\right\}$ is a list of complex numbers with $\lambda_{i} \in \mathcal{H}=\{z \in \mathbb{C}: \operatorname{Re} z \leq 0\}, \Lambda=\bar{\Lambda}, \lambda_{1} \geq\left|\lambda_{i}\right|, i=2, \ldots, n$, then $\Lambda$ is realizable if and only if

$$
s_{1}=\sum_{i=1}^{n} \lambda_{i} \geq 0, \quad s_{2}=\sum_{i=1}^{n} \lambda_{i}^{2} \geq 0, \quad s_{1}^{2} \leq n s_{2} .
$$

Now we show that a realizable list of complex numbers in the left-half plane is in particular realizable by a Toeplitz matrix.

Corollary 2.2. Let $\Lambda=\left\{\lambda_{1}, \lambda_{2}, \ldots, \lambda_{n}\right\}$, with $\operatorname{Re} \lambda_{i} \leq 0, i=2, \ldots, n$, be a realizable list of complex numbers. Then $\Lambda$ is also realizable by a Toeplitz matrix.

Proof. If $\Lambda$ is realizable, then from the result in [7], $\Lambda$ is the spectrum of a matrix of the form $\mathcal{C}+\alpha I$, where $\mathcal{C}$ is a nonnegative companion matrix with $\operatorname{tr}(\mathcal{C})=0, \alpha \geq 0$. Then, from Theorem 2.1 and Theorem 2.2, $\mathcal{C}$ is similar to a nonnegative Toeplitz matrix $T$ with eigenvalues $\lambda_{1}-\alpha, \lambda_{2}-\alpha, \ldots, \lambda_{n}-\alpha$. Hence $T+\alpha I$ is a nonnegative Toeplitz matrix with spectrum $\Lambda$.

Example 2.3. Let $\Lambda=\{13,-2+3 i,-2-3 i,-2+3 i,-2-3 i\}$. We construct a nonnegative Toeplitz matrix with spectrum $\Lambda$. Then we define

$$
\Gamma=\Lambda-\frac{\sum \lambda_{i}}{n}=\{12,-3+3 i,-3-3 i,-3+3 i,-3-3 i\}
$$

and compute the companion matrix of the polynomial

$$
\begin{aligned}
P(x) & =(x-12)(x+3-3 i)^{2}(x+3+3 i)^{2} \\
& =x^{5}-72 x^{3}-648 x^{2}-2268 x-3888,
\end{aligned}
$$

that is,

$$
\mathcal{C}=\left[\begin{array}{ccccc}
0 & 1 & 0 & 0 & 0 \\
0 & 0 & 1 & 0 & 0 \\
0 & 0 & 0 & 1 & 0 \\
0 & 0 & 0 & 0 & 1 \\
3888 & 2268 & 648 & 72 & 0
\end{array}\right]
$$

Now, from Theorem 2.1 and (6), we have the nonnegative Toeplitz matrix

$$
T^{\prime}=\left[\begin{array}{ccccc}
0 & 1 & 0 & 0 & 0 \\
18 & 0 & 1 & 0 & 0 \\
216 & 18 & 0 & 1 & 0 \\
1620 & 216 & 18 & 0 & 1 \\
11664 & 1620 & 216 & 18 & 0
\end{array}\right]
$$

with spectrum $\Gamma$. Then $T=T^{\prime}+I$ is the required nonnegative Toeplitz matrix. 
Example 2.4. Consider $\Lambda=\{14,-2,-2,-1,-1,-1 \pm 2 i,-2 \pm 3 i,-1 \pm 3 i\}$. The characteristic polynomial associated with $\Lambda$ is

$$
\begin{aligned}
p(x)= & x^{11}-87 x^{9}-964 x^{8}-5779 x^{7}-23492 x^{6}-68857 x^{5} \\
& -146580 x^{4}-223686 x^{3}-230612 x^{2}-139640 x-36400,
\end{aligned}
$$

and then the corresponding companion matrix is nonnegative. By using the MATLAB routine presented in the Appendix, Section 5, we compute the entries $t_{k}$ of our nonnegative Toeplitz matrix $T$ with spectrum $\Lambda$ :

$$
\begin{aligned}
t_{2} & =\frac{87}{10}, t_{3}=\frac{964}{9}, t_{4}=\frac{53149}{50}, t_{5}=\frac{162164}{15}, \\
t_{6} & =\frac{4463925637}{40500}, t_{7}=\frac{82990237}{75}, t_{8}=\frac{2335231077941}{216000} \\
t_{9} & =\frac{27636959655994}{273375}, t_{10}=\frac{3172986740312171}{3600000}, \\
t_{11} & =\frac{24712157597057887}{3645000} .
\end{aligned}
$$

In [9, Theorem 3] it was shown that the construction of the unit upper Hessenberg Toeplitz matrix is unique. Then, since our realizing Toeplitz matrices are unit lower Hessenberg Toeplitz, they are the same as the transpose of the unit upper Hessenberg Toeplitz matrices constructed in [9]. Therefore, our construction is also unique. However, our interest is the construction of nonnegative Toeplitz matrices with prescribed spectrum, which in our case become nonnegative unit lower Hessenberg Toeplitz ones.

Since the characteristic and minimal polynomials of companion matrices are equal, the following result is immediate:

Corollary 2.3. The Toeplitz matrices obtained from Theorem 2.1 have Jordan canonical form with only one Jordan block for each distinct eigenvalue.

\section{Symmetric nonnegative block Toeplitz matrices with prescribed Jordan canonical form}

We say that a spectrum is universally realizable (UR) if it is realizable for every possible Jordan canonical form $(J C F)$ allowed by the spectrum. In this Section, we explore the universal realizability of lists which are realizable by symmetric nonnegative block Toeplitz matrices (they are not necessarily symmetric). Our results give sufficient conditions for the existence of a symmetric nonnegative block Toeplitz matrix with prescribed JCF. First, we give some conditions for the existence of a symmetric nonnegative Toeplitz matrix with prescribed spectrum $\Lambda=\left\{\lambda_{1}, \ldots, \lambda_{n}\right\}$ for $n=2,3$. For $n=2$ we have that $\Lambda=\left\{\lambda_{1}, \lambda_{2}\right\}$ with $\lambda_{1} \geq\left|\lambda_{2}\right|, \lambda_{1}>0$, is the spectrum of the symmetric nonnegative Toeplitz matrix

$$
A=\frac{1}{2}\left[\begin{array}{cc}
\lambda_{1}+\lambda_{2} & \lambda_{1}-\lambda_{2} \\
\lambda_{1}-\lambda_{2} & \lambda_{1}+\lambda_{2}
\end{array}\right] .
$$

For $n=3$, we use a well known result due to Fiedler [4], which establishes that the real numbers $\lambda_{1} \geq \lambda_{2} \geq$ $\lambda_{3}$ and $a_{1} \geq a_{2} \geq a_{3} \geq 0$ are, respectively, the eigenvalues and the diagonal entries of a $3 \times 3$ symmetric nonnegative matrix if and only if the vector of eigenvalues $\left[\lambda_{1}, \lambda_{2}, \lambda_{3}\right]$ majorizes the vector of diagonal entries $\left[a_{1}, a_{2}, a_{3}\right]$ and $a_{1} \geq \lambda_{2}$. Then, for our purpose, we have: 
Lemma 3.1. The real numbers $\lambda_{1} \geq \lambda_{2} \geq \lambda_{3}$, and $a \geq 0$, are the eigenvalues and the diagonal entries, respectively, of a symmetric nonnegative Toeplitz matrix if and only if

$$
\left.\begin{array}{c}
\lambda_{1} \geq a \\
\lambda_{1}+\lambda_{2} \geq 2 a \\
\lambda_{1}+\lambda_{2}+\lambda_{3}=3 a \\
\lambda_{2} \leq a .
\end{array}\right\}
$$

In [17, Remark 3.3], the authors construct a $3 \times 3$ symmetric nonnegative matrix, which, in our case, after a row-column permutation, becomes the symmetric nonnegative Toeplitz matrix

$$
T=\left[\begin{array}{ccc}
a & \frac{s}{\sqrt{2}} & \alpha-a \\
s & a & \frac{s}{\sqrt{2}} \\
\alpha-a & \frac{s}{\sqrt{2}} & a
\end{array}\right],
$$

with spectrum $\Lambda=\left\{\lambda_{1}, \lambda_{2}, \lambda_{3}\right\}$, where $\alpha=\lambda_{1}+\lambda_{2}-a$ and $s=\sqrt{\left(\lambda_{1}-\alpha\right)\left(\lambda_{1}-a\right)}$, or the symmetric nonnegative Toeplitz matrix

$$
T^{\prime}=\left[\begin{array}{ccc}
a & \frac{t}{\sqrt{2}} & \beta-a \\
\frac{t}{\sqrt{2}} & a & \frac{t}{\sqrt{2}} \\
\beta-a & \frac{t}{\sqrt{2}} & a
\end{array}\right],
$$

with spectrum $\Lambda=\left\{\lambda_{1}, \lambda_{2}, \lambda_{3}\right\}$, where $\beta=\lambda_{1}+\lambda_{3}-a$ and $t=\sqrt{\left(\lambda_{1}-\beta\right)\left(\lambda_{1}-a\right)}$.

For real lists of the form $\Lambda=\left\{\lambda_{1}, \lambda_{2}, \ldots, \lambda_{2}\right\}$, we have the following proposition:

Proposition 3.1. Let $\Lambda=\left\{\lambda_{1}, \lambda_{2}, \ldots, \lambda_{2}\right\}$ be a list of $n$ real numbers with $\lambda_{2}<0$ and $\lambda_{1}+(n-1) \lambda_{2} \geq 0$. Then $\Lambda$ is the spectrum of a symmetric nonnegative Toeplitz matrix.

Proof. Without loss of generality we assume that $\lambda_{1}+(n-1) \lambda_{2}=0$. Consider the initial matrix

$$
B=\left[\begin{array}{ccccc}
\lambda_{1} & 0 & \cdots & \cdots & 0 \\
\lambda_{1}-\lambda_{2} & \lambda_{2} & 0 & & \vdots \\
\vdots & 0 & \ddots & \ddots & \vdots \\
\vdots & \vdots & \ddots & \ddots & 0 \\
\lambda_{1}-\lambda_{2} & 0 & \cdots & 0 & \lambda_{2}
\end{array}\right] \in \mathcal{C S}_{\lambda_{1}} .
$$

Let $\mathbf{q}^{T}=\left[(n-1) \lambda_{2}, \quad-\lambda_{2}, \cdots,-\lambda_{2}\right]$. Then from a well known result due to Brauer [1], $A=B+\mathbf{e q} \mathbf{q}^{T}$ is the required symmetric nonnegative Toeplitz matrix.

Let $A$ be an $n \times n$ matrix with spectrum $\Lambda=\left\{\lambda_{1}, \ldots, \lambda_{n}\right\}$, and let $X$ be the $n \times r$ matrix, $r<n$, whose columns $x_{i}$, $\left\|x_{i}\right\|=1, i=1, \ldots, r$, are eigenvectors of $A$ corresponding to the eigenvalues $\lambda_{1}, \ldots, \lambda_{r}$, with $\operatorname{rank}(X)=r$. Let $B=\Omega+C$ be an $r \times r$ symmetric nonnegative matrix with spectrum $\Lambda_{0}=\left\{\mu_{1}, \ldots, \mu_{r}\right\}$. Let $\Omega=\operatorname{diag}\left\{\lambda_{1}, \ldots, \lambda_{r}\right\}$ and let $S$ be an $n \times n$ orthogonal matrix partitioned as $S=[X \mid Y]$ with $S^{-1}=\left[\frac{X^{T}}{Y^{T}}\right]$. Then, since $A X=X \Omega$, we have

$$
\begin{aligned}
S^{-1} A S & =\left[\begin{array}{cc}
\Omega & X^{T} A Y \\
0 & Y^{T} A Y
\end{array}\right], S^{-1} X C X^{T} S=\left[\begin{array}{ll}
C & 0 \\
0 & 0
\end{array}\right] \\
S^{-1}\left(A+X C X^{T}\right) S & =\left[\begin{array}{cc}
B & X^{T} A Y \\
0 & Y^{T} A Y
\end{array}\right] .
\end{aligned}
$$


Now, from a result in [12, Chapter VI, Lemma 1.2], if the matrices $B$ and $Y^{T} A Y$ in (19) have no common eigenvalues, then $J\left(A+X C X^{T}\right)=J(B) \oplus J\left(Y^{T} A Y\right)$.

Next, we apply a perturbation result given in [17], which introduces a symmetric version of a result due to Rado and published by Perfect in [13]. Rado's result is a generalization of a Brauer's result [1] and it shows how to change $r$ eigenvalues of a matrix without changing any of its remaining $(n-r)$ eigenvalues (see $[16,17]$ and $[2,18]$, respectively, about how Rado's result has been applied to the NIEP and to the universal realizability problem). Thus we have:

Theorem 3.1. Let $\Lambda=\left\{\lambda_{1}, \lambda_{2}, \ldots, \lambda_{n}\right\}$ be a list of complex numbers. Suppose that:

i) There exists a partition $\Lambda=\Lambda_{0} \cup \underbrace{\Lambda_{1} \cup \cdots \cup \Lambda_{1}}_{\text {rtimes }}$, where

$$
\begin{aligned}
& \Lambda_{0}=\left\{\lambda_{01}, \lambda_{02}, \ldots, \lambda_{0 r}\right\} \text { is a real list } \\
& \Lambda_{1}=\left\{\lambda_{11}, \lambda_{12}, \ldots, \lambda_{1 p}\right\}, \Lambda_{0} \cap \Lambda_{1}=\varnothing,
\end{aligned}
$$

such that $\Gamma_{1}=\left\{\lambda, \lambda_{11}, \lambda_{12}, \ldots, \lambda_{1 p}\right\}, 0 \leq \lambda \leq \lambda_{1}$, is the spectrum of $a(p+1) \times(p+1)$ nonnegative matrix with certain JCF.

ii) There exists an $r \times r$ symmetric nonnegative Toeplitz matrix with spectrum $\Lambda_{0}$ and diagonal entries $\lambda, \lambda, \ldots, \lambda$ ( $r$ times).

Then, there exists a symmetric nonnegative block Toeplitz matrix $T$ with spectrum $\Lambda$ and JCF preserving the JCF of $\Lambda_{1}$.

Proof. From $i$ ) let $A_{1}$ be a $(p+1) \times(p+1)$ nonnegative matrix with spectrum $\Gamma_{1}$ and with certain prescribed $J C F$. Then, let $A$ be the $r(p+1) \times r(p+1)$ block diagonal matrix

$$
A=\left[\begin{array}{cccc}
A_{1} & & & \\
& A_{1} & & \\
& & \ddots & \\
& & & A_{1}
\end{array}\right] .
$$

Let $A_{1} \mathbf{x}=\lambda \mathbf{x}$ with $\|\mathbf{x}\|=1$, where $\lambda$ and $\mathbf{x}$ are the Perron eigenvalue and the Perron eigenvector of $A_{1}$, respectively. From ii) let $B$ be the $r \times r$ symmetric nonnegative Toeplitz matrix with spectrum $\Lambda_{0}$ and diagonal entries $\lambda, \lambda, \ldots, \lambda$ ( $r$ times). Let $\Omega$ be the $r \times r$ diagonal matrix $\Omega=\operatorname{diag}\{\lambda, \lambda, \ldots, \lambda\}$. Then for $C=B-\Omega$,

$$
C=\left[\begin{array}{cccc}
0 & c_{1} & \cdots & c_{r-1} \\
c_{1} & 0 & \ddots & \vdots \\
\vdots & \ddots & 0 & c_{1} \\
c_{r-1} & \cdots & c_{1} & 0
\end{array}\right] \text {, and } X=\left[\begin{array}{cccc}
\mathbf{x} & 0 & \cdots & 0 \\
0 & \mathbf{x} & \ddots & \vdots \\
\vdots & \ddots & \ddots & 0 \\
0 & \cdots & 0 & \mathbf{x}
\end{array}\right] \text {, }
$$

where $X$ is the $r(p+1) \times r$ matrix of normalized eigenvectors of $A$, it follows that

$$
X C X^{T}=\left[\begin{array}{cccc}
0 & c_{1} \mathbf{x} \mathbf{x}^{T} & \cdots & c_{r-1} \mathbf{x x}^{T} \\
c_{1} \mathbf{x x}^{T} & 0 & \ddots & \vdots \\
\vdots & \ddots & \ddots & c_{1} \mathbf{x x}^{T} \\
c_{r-1} \mathbf{x x}^{T} & \cdots & c_{1} \mathbf{x x}^{T} & 0
\end{array}\right]
$$

and from the symmetric Rado's result,

$$
T=A+X C X^{T}=\left[\begin{array}{cccc}
A_{1} & c_{1} \mathbf{x x}^{T} & \cdots & c_{r-1} \mathbf{x x}^{T} \\
c_{1} \mathbf{x x}^{T} & A_{1} & \ddots & \vdots \\
\vdots & \ddots & \ddots & c_{1} \mathbf{x x}^{T} \\
c_{r-1} \mathbf{x} \mathbf{x}^{T} & \cdots & c_{1} \mathbf{x x}^{T} & A_{1}
\end{array}\right]
$$


is a symmetric nonnegative block Toeplitz matrix with spectrum $\Lambda$. Since $\Lambda_{0} \cap \Lambda_{1}=\varnothing, T$ has a JCF, which preserves the $J C F$ of $\Lambda_{1}$.

Similar to Theorem 3.1, with obvious minor changes, the following result gives sufficient conditions for the existence and construction of a symmetric nonnegative block Toeplitz matrix with prescribed spectrum.

Theorem 3.2. Let $\Lambda=\left\{\lambda_{1}, \lambda_{2}, \ldots, \lambda_{n}\right\}$ be a list of complex numbers. Suppose that:

i) There exists a partition $\Lambda=\Lambda_{0} \cup \underbrace{\Lambda_{1} \cup \cdots \cup \Lambda_{1}}_{r \text { times }}$, where

$$
\begin{aligned}
& \Lambda_{0}=\left\{\lambda_{01}, \lambda_{02}, \ldots, \lambda_{0 r}\right\} \text { is a real list } \\
& \Lambda_{1}=\left\{\lambda_{11}, \lambda_{12}, \ldots, \lambda_{1 p}\right\}
\end{aligned}
$$

such that $\Gamma_{1}=\left\{\lambda, \lambda_{11}, \lambda_{12}, \ldots, \lambda_{1 p}\right\}, 0 \leq \lambda \leq \lambda_{1}$, is the spectrum of $a(p+1) \times(p+1)$ nonnegative matrix.

ii) There exists an $r \times r$ symmetric nonnegative Toeplitz matrix with spectrum $\Lambda_{0}$ and diagonal entries $\lambda, \lambda, \ldots, \lambda$ ( $r$ times).

Then, there exists a symmetric nonnegative block Toeplitz matrix $T$ with spectrum $\Lambda$.

Example 3.1. Consider the list $\Lambda=\{5,1,0,-2,-2,-2\}$ with the partition

$$
\Lambda_{0}=\{5,1,0\}, \Lambda_{1}=\{-2\}, \Gamma_{1}=\{2,-2\} .
$$

Then

$$
A=\left[\begin{array}{llllll}
0 & 2 & 0 & 0 & 0 & 0 \\
2 & 0 & 0 & 0 & 0 & 0 \\
0 & 0 & 0 & 2 & 0 & 0 \\
0 & 0 & 2 & 0 & 0 & 0 \\
0 & 0 & 0 & 0 & 0 & 2 \\
0 & 0 & 0 & 0 & 2 & 0
\end{array}\right], X=\left[\begin{array}{ccc}
\frac{1}{\sqrt{2}} & 0 & 0 \\
\frac{1}{\sqrt{2}} & 0 & 0 \\
0 & \frac{1}{\sqrt{2}} & 0 \\
0 & \frac{1}{\sqrt{2}} & 0 \\
0 & 0 & \frac{1}{\sqrt{2}} \\
0 & 0 & \frac{1}{\sqrt{2}}
\end{array}\right]
$$

From (17) we compute a symmetric nonnegative Toeplitz matrix

$$
B=\left[\begin{array}{ccc}
2 & \sqrt{\frac{3}{2}} & 2 \\
\sqrt{\frac{3}{2}} & 2 & \sqrt{\frac{3}{2}} \\
2 & \sqrt{\frac{3}{2}} & 2
\end{array}\right]
$$

with eigenvalues 5, 1, 0 and diagonal entries $2,2,2$. Then for $C=B-\operatorname{diag}\{2,2,2\}$,

$$
T=A+X C X^{T}=\left[\begin{array}{cccccc}
0 & 2 & \frac{\sqrt{6}}{4} & \frac{\sqrt{6}}{4} & 1 & 1 \\
2 & 0 & \frac{\sqrt{6}}{4} & \frac{\sqrt{6}}{4} & 1 & 1 \\
\frac{\sqrt{6}}{4} & \frac{\sqrt{6}}{4} & 0 & 2 & \frac{\sqrt{6}}{4} & \frac{\sqrt{6}}{4} \\
\frac{\sqrt{6}}{4} & \frac{\sqrt{6}}{4} & 2 & 0 & \frac{\sqrt{6}}{4} & \frac{\sqrt{6}}{4} \\
1 & 1 & \frac{\sqrt{6}}{4} & \frac{\sqrt{6}}{4} & 0 & 2 \\
1 & 1 & \frac{\sqrt{6}}{4} & \frac{\sqrt{6}}{4} & 2 & 0
\end{array}\right]
$$

is a symmetric nonnegative block Toeplitz matrix with spectrum $\Lambda$.

It is easy to see that if $T$ is obtained from Theorem 3.1, then we have the following necessary condition:

Proposition 3.2. Let $T$ be an $n \times n$ symmetric nonnegative block Toeplitz matrix, obtained from Theorem 3.1, with spectrum $\Lambda=\left\{\lambda_{1}, \lambda_{2}, \ldots, \lambda_{n}\right\}$ and $r$ blocks of size $t(n=r t)$. Then $\Lambda$ has $r$ lists of $(t-1)$ repeated eigenvalues. 
Example 3.2. Consider the list

$$
\Lambda=\{9,5,4,-3+2 i,-3-2 i,-3+2 i,-3-2 i,-3+2 i,-3-2 i\}
$$

with the partition

$$
\Lambda_{0}=\{9,5,4\}, \Lambda_{1}=\{-3+2 i,-3-2 i\}, \Gamma_{1}=\{6,-3+2 i,-3-2 i\} .
$$

Then

$$
T_{1}=\left[\begin{array}{ccc}
0 & 1 & 0 \\
\frac{23}{2} & 0 & 1 \\
78 & \frac{23}{2} & 0
\end{array}\right] \text { and } B=\left[\begin{array}{ccc}
6 & \sqrt{\frac{3}{2}} & 2 \\
\sqrt{\frac{3}{2}} & 6 & \sqrt{\frac{3}{2}} \\
2 & \sqrt{\frac{3}{2}} & 6
\end{array}\right]
$$

have, respectively, the spectra $\Gamma_{1}$ and $\Lambda_{0}$. Moreover, $T_{1}$ has a normalized Perron eigenvector $\frac{2}{\sqrt{2549}}\left[1,6, \frac{49}{2}\right]$. Let $A=\operatorname{diag}\left\{T_{1}, T_{1}, T_{1}\right\}$. Then the matrix $T=A+X C X^{T}$,

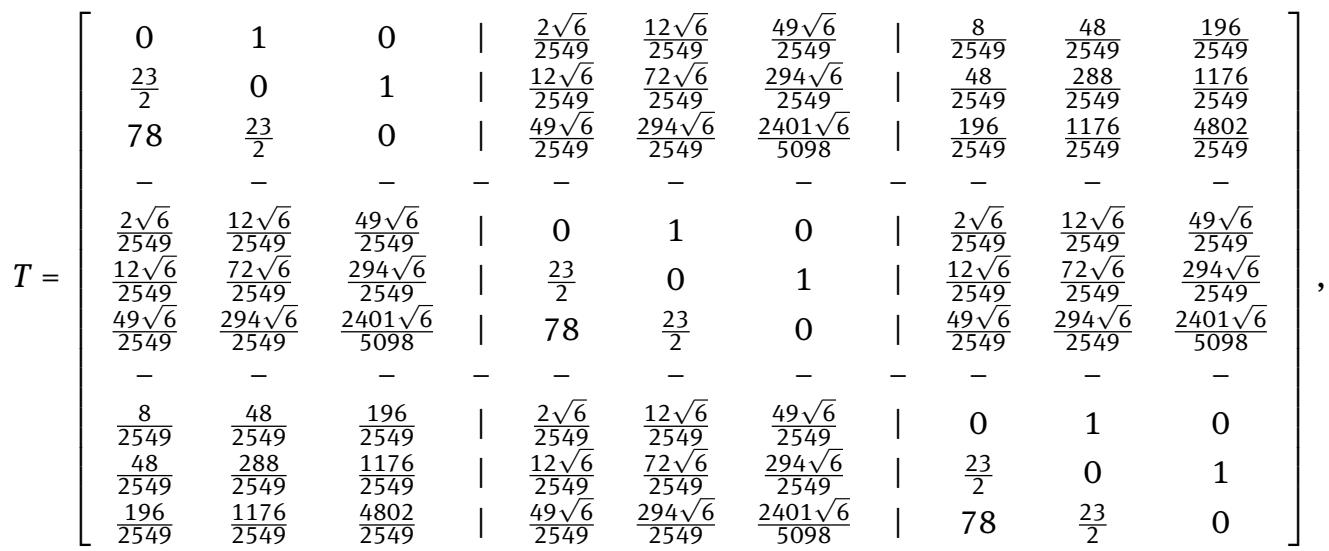

is a symmetric nonnegative block Toeplitz with spectrum $\Lambda$.

Example 3.3. Let $\Lambda=\{20,12,-3,-3,-3,-3,-5,-5,-5,-5\}$.

i) First we compute a block symmetric nonnegative Toeplitz matrix with spectrum $\Lambda$ and linear JCF. To do that we take the partition

$$
\begin{aligned}
\Lambda & =\Lambda_{0} \cup \Lambda_{1} \cup \Lambda_{1} \text { with } \\
\Lambda_{0} & =\{20,12\}, \Lambda_{1}=\{-3,-3,-5,-5\}, \Gamma_{1}=\{16,-3,-3-5,-5\} .
\end{aligned}
$$

Next, from the procedure in [15], we compute the nonnegative matrix

$$
A_{1}=\left[\begin{array}{lllll}
0 & 3 & 3 & 5 & 5 \\
3 & 0 & 3 & 5 & 5 \\
3 & 3 & 0 & 5 & 5 \\
5 & 3 & 3 & 0 & 5 \\
5 & 3 & 3 & 5 & 0
\end{array}\right]
$$

with spectrum $\Gamma_{1}$, Perron eigenvector $\mathbf{x}=\frac{1}{\|\mathbf{e}\|} \mathbf{e}$, and diagonal JCF. We also compute the symmetric nonnegative Toeplitz matrix

$$
B=\left[\begin{array}{cc}
16 & 4 \\
4 & 16
\end{array}\right] \text {, }
$$


with spectrum $\Lambda_{0}$ and constant diagonal entry 16 . Then

$$
\begin{aligned}
& T_{1}=A+X C X^{T} \\
& =\left[\begin{array}{ll}
A_{1} & \\
& A_{1}
\end{array}\right]+\left[\begin{array}{ll}
\mathbf{x} & 0 \\
0 & \mathbf{x}
\end{array}\right]\left[\begin{array}{ll}
0 & 4 \\
4 & 0
\end{array}\right]\left[\begin{array}{cc}
\mathbf{x}^{T} & 0 \\
0 & \mathbf{x}^{T}
\end{array}\right] \\
& =\left[\begin{array}{llllllllll}
0 & 3 & 3 & 5 & 5 & \frac{4}{5} & \frac{4}{5} & \frac{4}{5} & \frac{4}{5} & \frac{4}{5} \\
3 & 0 & 3 & 5 & 5 & \frac{4}{5} & \frac{4}{5} & \frac{4}{5} & \frac{4}{5} & \frac{4}{5} \\
3 & 3 & 0 & 5 & 5 & \frac{4}{5} & \frac{4}{5} & \frac{4}{5} & \frac{4}{5} & \frac{4}{5} \\
5 & 3 & 3 & 0 & 5 & \frac{4}{5} & \frac{4}{5} & \frac{4}{5} & \frac{4}{5} & \frac{4}{5} \\
5 & 3 & 3 & 5 & 0 & \frac{4}{5} & \frac{4}{5} & \frac{4}{5} & \frac{4}{5} & \frac{4}{5} \\
4 & 4 & 4 & 4 & 4 & 0 & 3 & 3 & 5 & 5 \\
5 & 5 & \frac{5}{5} & \frac{5}{5} & 0 & 3 & 3 & 5 \\
4 & \frac{4}{5} & \frac{4}{5} & \frac{4}{5} & \frac{4}{5} & 3 & 0 & 3 & 5 & 5 \\
\frac{4}{5} & \frac{4}{5} & \frac{4}{5} & \frac{4}{5} & \frac{4}{5} & 3 & 3 & 0 & 5 & 5 \\
4 & \frac{4}{5} & \frac{4}{5} & \frac{4}{5} & \frac{4}{5} & 5 & 3 & 3 & 0 & 5 \\
\frac{4}{5} & \frac{4}{5} & \frac{4}{5} & \frac{4}{5} & \frac{4}{5} & 5 & 3 & 3 & 5 & 0
\end{array}\right]
\end{aligned}
$$

is a symmetric nonnegative block Toeplitz matrix with spectrum $\Lambda$ and diagonal JCF.

ii) With the same partition we compute, from the procedure in [18], the nonnegative matrix

$$
A_{2}=\left[\begin{array}{lllll}
0 & 3 & 3 & 5 & 5 \\
4 & 0 & 2 & 5 & 5 \\
3 & 3 & 0 & 5 & 5 \\
6 & 3 & 3 & 0 & 4 \\
5 & 3 & 3 & 5 & 0
\end{array}\right]
$$

with $J C F J\left(A_{2}\right)=\operatorname{diag}\left\{J_{1}(16), J_{2}(-3), J_{2}(-5)\right\}$. Then

$$
T_{2}=\left[\begin{array}{ll}
A_{2} & \\
& A_{2}
\end{array}\right]+X C X^{T}
$$

is a symmetric nonnegative block Toeplitz matrix with spectrum $\Lambda$ and with $J C F \quad J\left(T_{2}\right)=$ $\operatorname{diag}\left\{J_{1}(20), J_{1}(12), J_{2}(-3), J_{2}(-3), J_{2}(-5), J_{2}(-5)\right\}$.

iii) In the same way, we may compute symmetric nonnegative block Toeplitz matrices $T_{3}$ and $T_{4}$ with Jordan blocks $J_{2}(-3), J_{2}(-3), J_{1}(-5), J_{1}(-5), J_{1}(-5), J_{1}(-5)$ and $J_{1}(-3), J_{1}(-3), J_{1}(-3), J_{1}(-3), J_{2}(-5), J_{2}(-5)$, respectively.

\section{Appendix}

In this Section, we present an algorithm that computes matrices $\mathcal{C}$ and $T$ from Section 2 . The informed experiment in Example 2.4 was performed using Matlab R2017a on a PC with a $3.6 \mathrm{GHz}$ intel (R) Core (TM) i7 processor, with $8 \mathrm{~GB}$ of RAM and with operating system windows 10 Pro version 17134.829. The Partitions Function finds the indices $l_{2}, l_{3}, \ldots, l_{k-2}$ of the sum in (13) and it can be found in [3].

function $[\mathrm{T}, \mathrm{C}]=$ comptoep (varargin)

$\%$ Algorithm to construct similar matrices $\mathrm{C}$ and $\mathrm{T}$, where $\mathrm{C}$ is a companion

$\%$ matrix and $\mathrm{T}$ is a Toeplitz matrix such that eig(C)=eig( $\mathrm{T})$

$\%$ for a given set of eigenvalues defined by the user.

$\%$ INPUT

$\%$ - varargin is a variable-length input argument list that contains the

$\%$ eigenvalues defined by the user as lambda_1, \lambda_2,.., \lambda_n. 
$\%$

$\%$ OUTPUT

$\%-\mathrm{C}$ is a companion matrix whose eigenvalues are the list defined $\%$ by the user.

$\%$ - $\mathrm{T}$ is a Toeplitz matrix which is similar to C.

\% Author: Javier G. Pizarro

$\%$ e-mail: javier.gonzalez01@ce.ucn.cl

$\%$ Release date: 6/24/19

$\mathrm{n}=$ length (varargin); C=zeros $(\mathrm{n}, \mathrm{n})$;

in=cell2mat (varargin);

syms $\mathrm{x}$

$\%$ Constructing $\mathrm{C}$

$c c=\operatorname{expand}(\operatorname{prod}(x-i n))$;

$\mathrm{cc}=\operatorname{sym} 2 \mathrm{poly}(\mathrm{cc})$;

$\mathrm{C}(\mathrm{n},:)=-\mathrm{cc}(\mathrm{n}+1:-1: 2)$;

$C(1: n-1,2: n)=$ eye $(n-1)$;

$\%$ Start the proccess for constructing $\mathrm{T}$

$c c=-c c(2: n+1)$;

$\mathrm{T}=\operatorname{zeros}(\mathrm{n}, \mathrm{n})$;

$\mathrm{t}=\operatorname{zeros}(\mathrm{n}, 1)$;

$\mathrm{t}(2)=\mathrm{cc}(2) /(\mathrm{n}-1)$;

if $n==2$

$\mathrm{T}(2,1)=\mathrm{t}(2) ; \mathrm{T}(1,2)=1$;

return

end

$\mathrm{t}(3)=\mathrm{cc}(3) /(\mathrm{n}-2)$;

if $\mathrm{n}==3$

$\mathrm{T}(1,2)=1 ; \quad \mathrm{T}(2,3)=1 ;$

$\mathrm{T}(:, 1)=\mathrm{t} ; \mathrm{T}(3,2)=\mathrm{t}(2)$;

return

end

$\%$ For $n>=4$ start the computation of li's $\% \%$

for $i=4: n$

coef=partitions (i, 2:i-2);

[ncomb, nvar] =size (coef);

for $k=1: n c o m b$

produ $=1$;

for $1=1$ :nvar

produ=produ* $(-t(1+1))^{\wedge} \operatorname{coef}(k, 1)$;

end

$u=n-i ; n u m=f a c t o r i a l(u+n o r m(\operatorname{coef}(k,:), 1))$;

den=factorial (u);

for $1=1$ :nvar

den=den $*$ factorial $(\operatorname{coef}(k, 1))$;

end

$t(i)=t(i)+$ num $*$ produ $/$ den;

end

$t(i)=(t(i)+c c(i)) /(n-i+1)$;

end 


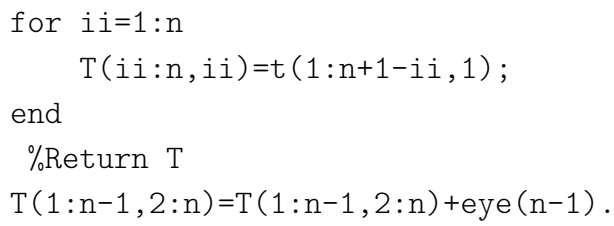

Acknowledgement: Supported by Fondecyt 1170313, Chile, Conicyt-PCHA/Doc.Nac./2016-21160684, Chile, and Universidad Católica del Norte.

\section{References}

[1] A. Brauer, Limits for the characteristic roots of a matrix IV. Applications to stochastic matrices. Duke Math. J. 19 (1952) 75-91.

[2] M. Collao, C. R. Johnson, R. L. Soto, Universal realizability of spectra with two positive eigenvalues, Linear Algebra Appl. 545 (2018) 226-239.

[3] J. D’Errico, Partitions of an integer, MathWorks ${ }^{\circledR}$ https://la.mathworks.com/matlabcentral/fileexchange/12009-partitionsof-an-integer (2018).

[4] M. Fiedler, Eigenvalues of nonnegative symmetric matrices, Linear Algebra Appl. 9 (1974) 119-142.

[5] A.I. Julio, R.L. Soto, Persymmetric and bisymmetric nonnegative inverse eigenvalue problem, Linear Algebra Appl. 469 (2015) 130-152.

[6] T. J. Laffey, M. E Meehan, A characterization of trace zero nonnegative $5 \times 5$ matrices, Linear Algebra Appl. $302-303$ (1999) 295-302.

[7] T.J. Laffey, H. Šmigoc, Nonnegative realization of spectra having negative real parts, Linear Algebra Appl. 416 (2006) 148-159.

[8] R. Loewy, D. London, A note on the inverse problem for nonnegative matrices, Linear Multilinear Algebra 6 (1978) 83-90.

[9] D.S. Mackey, N. Mackey, S. Petrovic, Is every matrix similar to a Toeplitz matrix?, Linear Algebra Appl. 297 (1999) 87-105.

[10] M. E. Meehan, Some results on matrix spectra, Ph. D. Thesis, National University of Ireland, Dublin, 1998.

[11] M. Merca, A note on the determinant of a Toeplitz-Hessenberg matrix, Spec. Matrices 1 (2013) 10-16

[12] H. Minc, Nonnegative matrices, John Wiley \& sons, New York, 1988.

[13] H. Perfect, Methods of constructing certain stochastic matrices II, Duke Math. J. 22 (1955) 305-311.

[14] H. Šmigoc, The inverse eigenvalue problem for nonnegative matrices, Linear Algebra Appl. 393 (2004) 365-374.

[15] R. L. Soto, Existence and construction of nonnegative matrices with prescribed spectrum, Linear Algebra Appl. 369 (2003) 169-184.

[16] R.L. Soto, O. Rojo, Applications of a Brauer theorem in the nonnegative inverse eigenvalue problem, Linear Algebra Appl. 416 (2006) 844-856.

[17] R.L. Soto, O. Rojo, J. Moro, A. Borobia, Symmetric nonnegative realization of spectra, Electron. J. Linear Algebra 16 (2007) 1-18.

[18] R.L. Soto, R.C. Diaz, H. Nina, M. Salas, Nonnegative matrices with prescribed spectrum and elementary divisors, Linear Algebra Appl. 439 (2013) 3591-3604.

[19] R.L. Soto, A.I. Julio, M. Salas, Nonnegative persymmetric matrices with prescribed elementary divisors, Linear Algebra Appl. 483 (2015) 130-157.

[20] H.R. Suleimanova, Stochastic matrices with real characteristic values, Dokl. Akad. Nauk SSSR 66 (1949) 343-345.

[21] J. Torre-Mayo, M. R. Abril-Raimundo, E. Alarcia-Estévez, C. Marijuán, M. Pisonero, The nonnegative inverse eigenvalue problem from the coefficients of the characteristic polynomial. EBL digraphs, Linear Algebra Appl. 426 (2007) 729-773. 